\title{
UTILITY POLES EXTRACTION FROM MOBILE LIDAR DATA IN URBAN AREA BASED ON DENSITY INFORMATION
}

\author{
D. Shokri ${ }^{1}$, H. Rastiveis ${ }^{1, *}$, A. Shams ${ }^{2}$, W. A. Sarasua ${ }^{3}$ \\ ${ }^{1}$ Department of Photogrammetry and Remote Sensing, School of Surveying and Geospatial Engineering, College of Engineering, \\ University of Tehran, Tehran, Iran, (daneshshokri72, hrasti)@ut.ac.ir \\ ${ }^{2}$ Advanced Highway Maintenance \& Construction Technology (AHMCT) Research Centre, University of California, Davis, CA, \\ USA, asham@ucdavis.edu \\ ${ }^{3}$ Glenn Department of Civil Engineering, Clemson University, Clemson, SC, USA. sarasua@ clemson.edu
}

Commission II, WG II/10

KEY WORDS: Utility Poles, Mobile Terrestrial Laser Scanner, Point Clouds, Density Information, Hough Transform, LiDAR

\begin{abstract}
:
Utility poles located along roads play a key role in road safety and planning as well as communications and electricity distribution. In this regard, new sensing technologies such as Mobile Terrestrial Laser Scanner (MTLS) could be an efficient method to detect utility poles and other planimetric objects along roads. However, due to the vast amount of data collected by MTLS in the form of a point cloud, automated techniques are required to extract objects from this data. This study proposes a novel method for automatic extraction of utility poles from the MTLS point clouds. The proposed algorithm is composed of three consecutive steps of pre-processing, cable area detection, and poles extraction. The point cloud is first pre-processed and then candidate areas for utility poles are specified based on Hough Transform (HT). Utility poles are extracted by applying horizontal and vertical density information to these areas. The performance of the method was evaluated on a sample point cloud and $98 \%$ accuracy was achieved in extracting utility poles using the proposed method.
\end{abstract}

\section{INTRODUCTION}

A utility distribution network is vital for transferring electricity from power plants to power consumables (Guan et al., 2016). Mapping a utility network including poles and cables is vital due to the importance of electricity in modern life. Various technologies and equipment such as optical cameras (Ye et al., 2014), laser scanners (Bae et al., 2014), and satellite data (Yu et al., 2015a) have been proposed for mapping utility poles. There are a number of considerations when choosing a method for utility pole mapping including accuracy, safety, cost, and time. (Baffour, 2002; Shams et al., 2018; Souleyrette et al., 2003). High resolution aerial images can be accurate data sources, but manual extraction of pole locations is time consuming and conventional ground control surveying is needed to achieve high accuracy (Wen et al., 2019). Further, weather conditions can affect accuracy (Holopainen et al., 2013). Conventional surveying is especially tedious for large areas and poses safety risks to personnel due to their proximity to traffic (Shams et al., 2018; Souleyrette et al., 2003).

Recently, the use of vehicle mounted MTLS systems has been growing for utility mapping studies (Li et al., 2019a). MTLS systems are capable of collecting dense point clouds that have many applications including transportation infrastructure mapping, asset management, Geographic Information System (GIS) data collection, and three dimensional (3D) modelling (Shams et al., 2018). An MTLS system includes a high accuracy Global Positioning System (GPS), an Inertial Measurement Unit
(IMU), one or more video logging cameras, and a Distance Measuring Instrument (DMI) that is used to dead reckon between GPS points. (see Figure 1)(Che et al., 2019; Cui et al., 2019). Transportation agencies are willing to use MTLS for a number of applications because collecting accurate point cloud data can be collected at highway speed. These applications include road surface modeling and collection of traffic signs, poles, trees, and other planimetric features in or near a roadway's right-of-way. (Balado et al., 2017; Cabo et al., 2014; Chen et al., 2018; Riveiro et al., 2015; Serna and Marcotegui, 2014; Shams et al., 2018; Xu et al., 2018; Yadav and Chousalkar, 2017; Yang et al., 2015; Yang et al., 2012). In addition, ground based MTLS collection of utility poles adjacent to the roadway results in a denser dataset compared to Airborne Laser Scanning (ALS)(Li et al., 2019a).

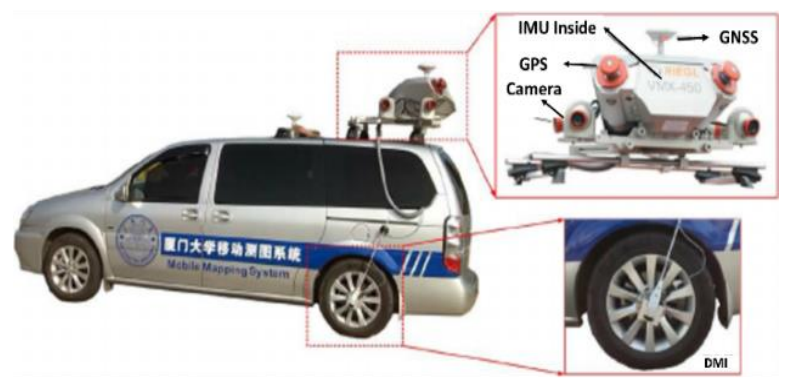

Figure 1. The MTLS technology with its sensors(Yu et al., 2015b)

* Corresponding author 
It is estimated that there billions of utility poles in use worldwide are more than 160 million utility poles in the United States alone. Many of these poles are adjacent to roads. An accurate inventory of utility poles for asset management and roadside safety planning is beneficial to transportation and public works agencies. The primary objective of this research is the development of an efficient framework for the automatic extraction of an accurate utility pole inventory from an MTLS point cloud. In the next section, we discuss related studies and how they influence this research. We follow with a discussion of the proposed method, experimental results, and conclusions.

\section{RELATED WORKS}

Utility pole mapping from point clouds can be categorized in two main groups: supervised and unsupervised classification. Supervised classification uses common classifiers such as linear discriminant analysis and Support Vector Machines (SVM) (Ordóñez et al., 2017; Wen et al., 2019) to extract interested objects. Although they can be accurate, their training processes are time consuming and require a large amount of manually collected training data to ensure accuracy (Che et al., 2019; Lehtomäki et al., 2019). Unsupervised classification methods usually focus on the cylindrical shape of poles (Hao et al., 2018; Landa and Ondroušek, 2016). These methods can potentially map pole-like objects located throughout diverse regions with no need for training data (Li et al., 2019b).

Numerous studies have focused on unsupervised classification of pole-like objects from point clouds collected by different LiDAR platforms (Cabo et al., 2014; Yadav et al., 2016; Yokoyama et al., 2011). However, few studies have focused on extracting only utility poles. In this regard, Lehtomäki et al., (2010) presented a scan line-based algorithm to map pole-shape objects such as traffic signs, lampposts and trees. They first segmented consecutive points based on scan line information. Then, due to the fact that poles are circular, any clusters that followed a circular form were considered to be poles. Their method was tested on a point cloud of a road environment collected using MTLS with $81.0 \%$ accuracy. Similarly, Yokoyama et al., (2011) proposed an algorithm to extract pole-shape objects based on three phases: Laplacian smoothing, Principal Component analysis (PCA), and detecting the degree of the pole-shape objects. They achieved an accuracy of $97.4 \%$. In the last decade, voxels-based methods and PCA have been frequently used to recognize the poles (Cabo et al., 2014; Kang et al., 2018; Yang et al., 2015). Likewise, Li et al., (2018) used PCA in their polelike objects classification method.

\section{METHODOLOGY}

The literature review has indicated that there is a need for a framework that can efficiently extract specific pole types - in our case, utility poles. It is proposed that by leveraging cable information from a LiDAR point cloud, utility poles can be distinguished from other types of poles. The workflow of the proposed method is shown in Figure 2 and extracts utility poles in three main steps:

1) Point cloud preprocessing including sectioning to reduce point cloud volume and speed-up implementation; noise removal; and low-height filtering to remove ground points and objects like bushes, and cars;

2) Cable area detection using the Hough Transform (HT) algorithm to estimate the search area for utility poles.

3) Extracting poles using horizontal and vertical density process.

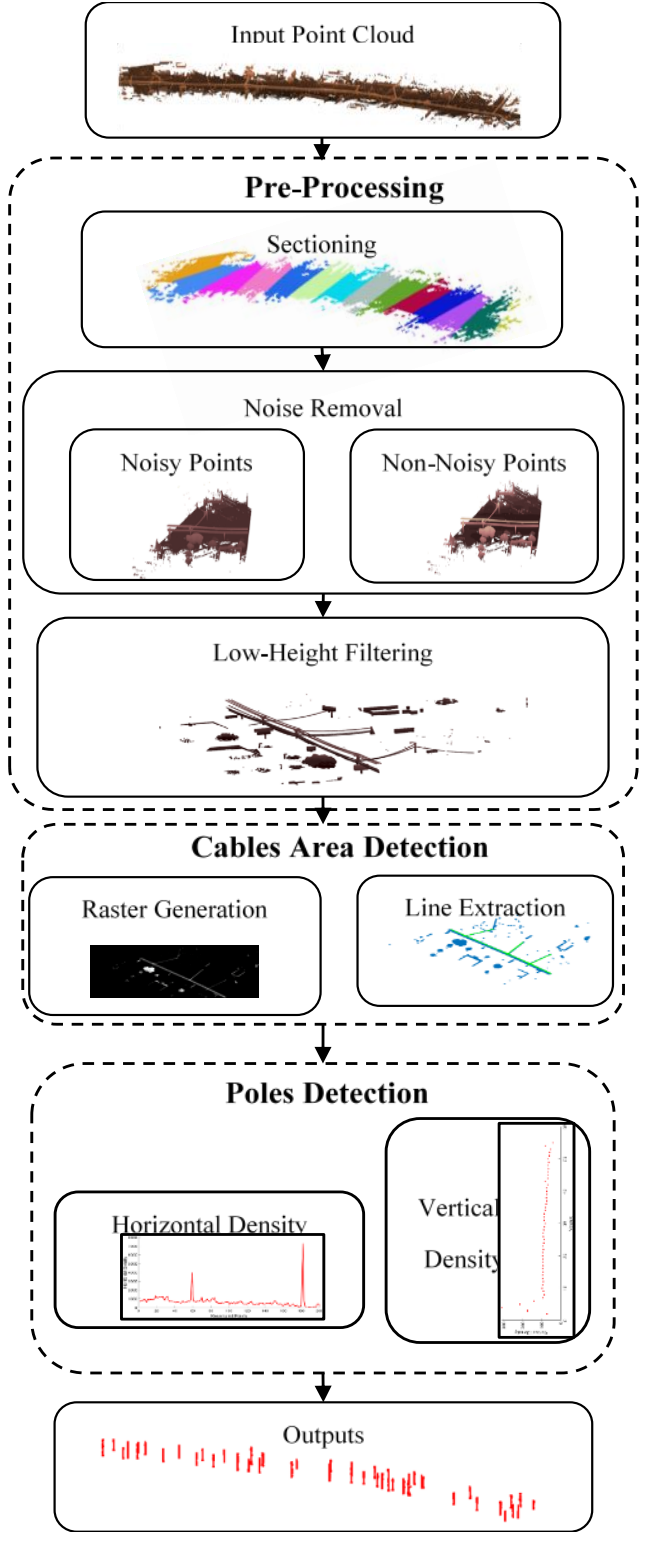

Figure 2. The flowchart of the proposed algorithm for poles extraction.

\subsection{Pre-Processing}

Dividing an MTLS point cloud into smaller sections will decrease overall processing time. The point cloud is divided into equal sections using the trajectory data (i.e. red lines in Figure 3). After sectioning, all additional processing is implemented on each individual section.

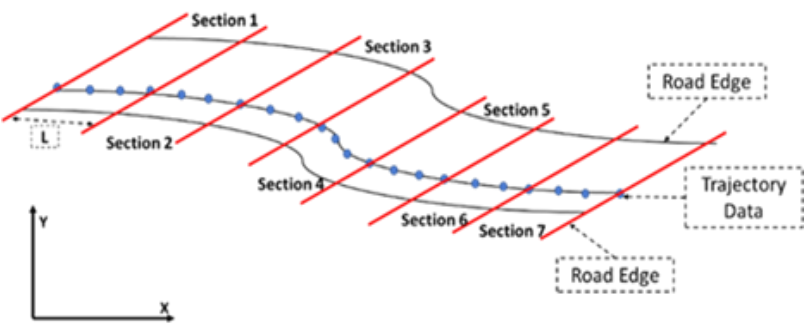

Figure 3. Presentation of the Pre-Processing step. 
After dividing the point clouds into small sections (e.g. tiles), the noisy points with abnormal altitude are deleted (see Figure 4) using the Sparse Outlier Removal method proposed by Rusu et al., (2008).

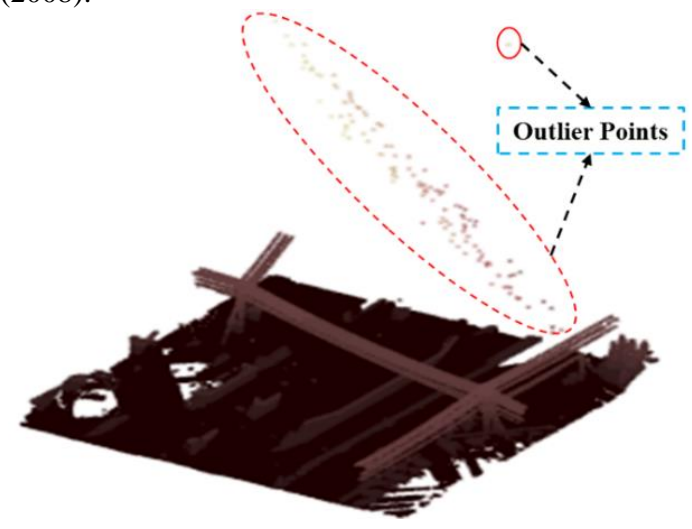

Figure 4. Identifying noisy points with abnormal altitude.

Using the Sparse Outlier Removal method, k-nearest neighbour $(\mathrm{k}-\mathrm{NN})$ points are selected around each single point. Then, the identifying elevation $(\mathrm{z})$ for noisy points, mean $(\mu)$ and standard deviation $(\sigma)$ as shown in Equation (1) and (2) are calculated. Finally, those points whose altitude falls within a specific range of the standard deviation (i.e. $\mu \pm \alpha \mathrm{S}$ where $\alpha$ is a coefficient for increasing impact of standard deviation) are considered as notnoisy $(N R)$ points (Equation 3 ) and all other points are removed.

$$
\begin{aligned}
& \mu=\frac{\left(z_{1}+z_{2}+\cdots z_{i}\right)}{k} \\
& \sigma=\left(\frac{\left[\left(z_{1}-\mu\right)^{2}+\left(z_{2}-\mu\right)^{2}+\cdots+\left(z_{i}-\mu\right)^{2}\right]}{k^{2}}\right) \\
& N R=\left\{\begin{array}{cc}
\text { Non }- \text { Noisy } & \mu-\alpha \times \sigma \leq z \leq \mu+\alpha \times \sigma \\
\text { Noisy } & \text { Otherwise }
\end{array}\right\}
\end{aligned}
$$

After the noise removal, the area along $\mathrm{X}$ and $\mathrm{Y}$ axis are partitioned into an $\mathrm{M} \times \mathrm{M}$ grid as shown in Figure 5. The minimum elevation within each cell is detected and then all points in the cells are shifted along the $\mathrm{Z}$ axis in which the minimum elevation would be zero. After that, a predefined threshold is considered to filter low-height objects.

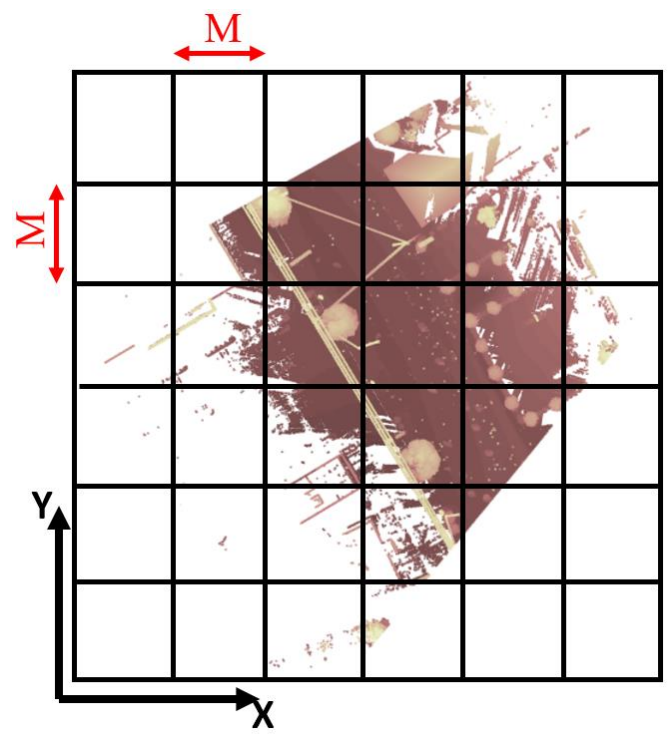

Figure 5. Method of eliminating low-height objects using gridding.

\subsection{Cable Area Detection}

Although many outliers are removed in the preprocessing step, lots of redundant points still remain in the point cloud that represent objects other than poles such as trees and buildings. Thus, searching for poles objects may still be tedious. In this study, the HT algorithm is used to bound the search area for utility pole extraction by detecting the wire paths. This algorithm is capable of detecting linear objects on binary images (Guan et al., 2016). For this purpose, a raster image is first created from the remaining points, and the HT algorithm is performed on the extracted binary edge image using an edge detection method (i.e. canny). A significant concept for the HT method is the mapping of single edge pixels on the binary image space to a line in the Hough space using Equation 4. The regions in Hough space where most lines intersect can be interpreted as true lines in the binary image.

$$
r=x \cos (\theta)+y \sin (\theta)
$$

In this research, the mathematical background of the HT algorithm is not discussed in detail and readers are referred to (Duda and Hart, 1972) for additional details of the HT algorithm.

\subsection{Poles Extraction}

After transferring the extracted lines from the image back to the point cloud space, candidate poles extraction (horizontal density process) and true poles identification (vertical density) are considered in order to detect poles. For the horizontal density process, resampled points are created on each extracted line. Afterwards, a rectangle with a length perpendicular to the line, is considered in order to calculate density. Using these densities for resampled points, a horizontal density diagram can be obtained. Next, the diagram is categorized into two groups of low- and high-density points using Otsu's thresholding method (Otsu, 1979). The high-density category is selected as the candidate for true poles identification.

A major problem with the selected candidate points is that denser objects other than poles can be extracted (e.g. trees). Therefore, vertical density stage is designed to identify true poles. For this purpose, first of all, same as the horizontal density process but along the Z-axis the density profile is obtained. It is assumed that a diagram of poles follows linear status whereas non-pole objects such as trees have a disordered case. Here, the true poles are those that the density of the sampling points are greater than zero, and also the calculated standard deviation of the density for all sampling points are less than a pre-defined threshold. This threshold can be found based on a number of training data.

\section{EXPERIMENTS AND RESULTS}

\subsection{Study Area}

This research was evaluated using MTLS data collected along a $750 \mathrm{~m}$ urban corridor in Anderson, South Carolina, USA. The study area includes various planimetric features such as utility poles, trees, traffic signs, and buildings. The yellow line in Figure 6-a displays the study area, and Figure 6-b shows the collected point cloud of this study area using the MTLS system.

\subsection{Results}

Due to the size of the point cloud (i.e. 1.4 Gigabytes), the sectioning step was done to generate smaller sections to reduce processing time. Figure 7 shows the resultant smaller sections using trajectory data with $60 \mathrm{~m}$ intervals. Figures $7-\mathrm{a}$ and $-\mathrm{b}$ show two sample sections of the point cloud. 


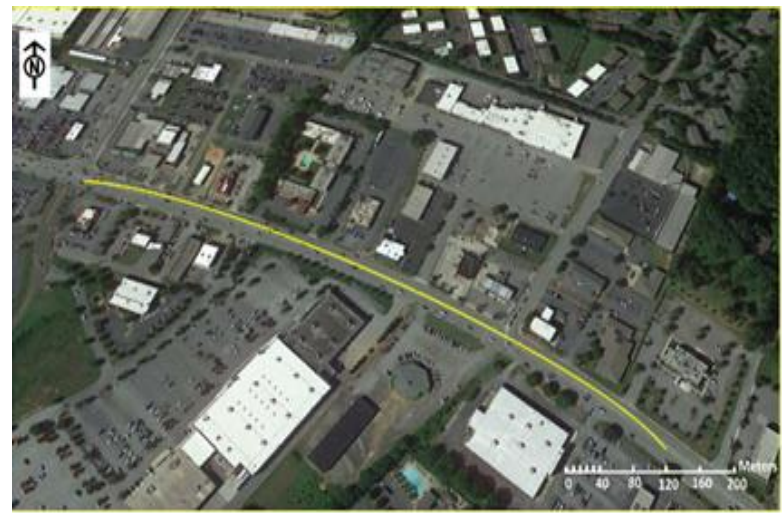

(a)

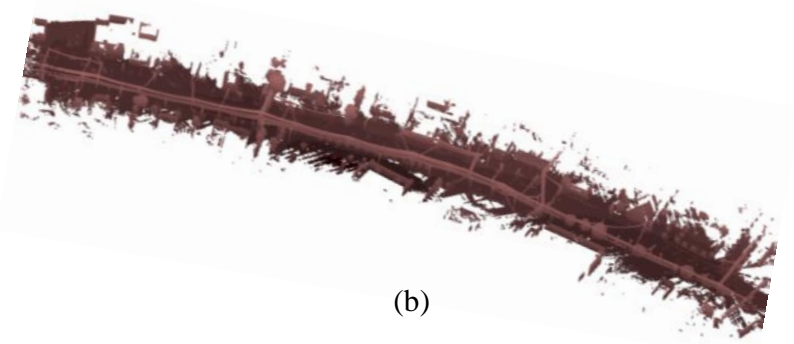

Figure 6. Study area: (a) Top view image of the study area; (b) Collected point clouds using the MTLS system.

After dividing the point clouds into smaller sections, noisy points were removed considering 20 neighborhood points and a coefficient equal to 1 . An elevation thematic map is shown in Figure 8-a where darker colors represent low altitude objects and brighter colors represent higher altitude objects. Figure 8-b, shows a maximum elevation change from $940 \mathrm{~m}$ to $840 \mathrm{~m}$ after removing noisy points.

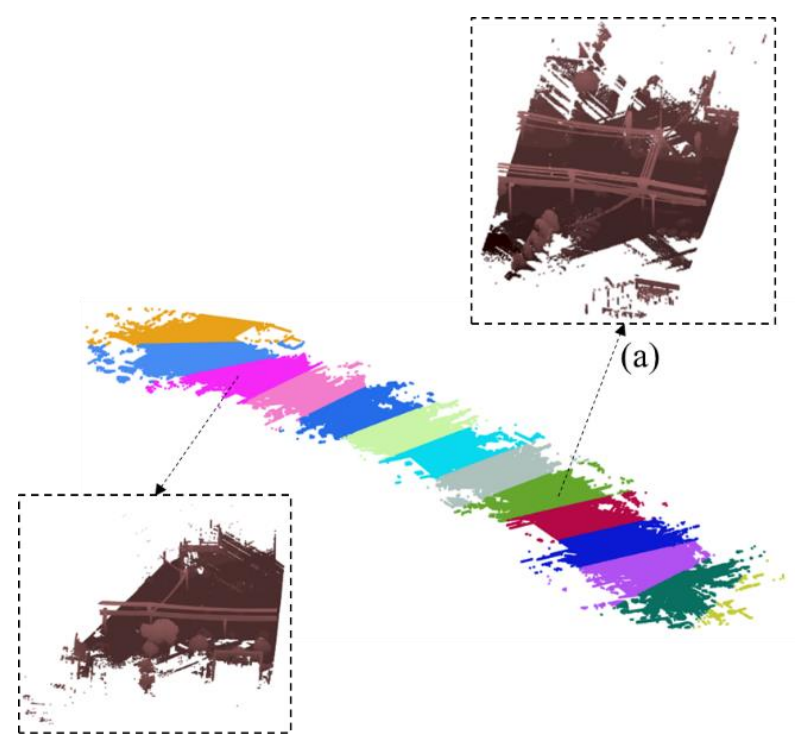

(b)

Figure 7. Sectioning of point cloud. (a) and (b) are two sample sections.

In the low-height objects filtering, $5 \times 5$ sq. m. cell size and $7 \mathrm{~m}$ threshold was considered. Figure 9-b shows the remaining points from a sample section after eliminating the low-height objects. As can be seen from this figure, the remaining points are mostly utility poles and cables.

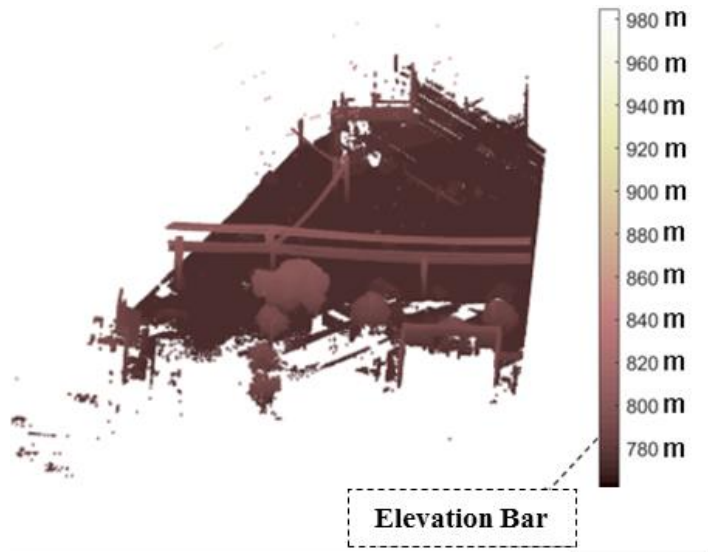

(a)

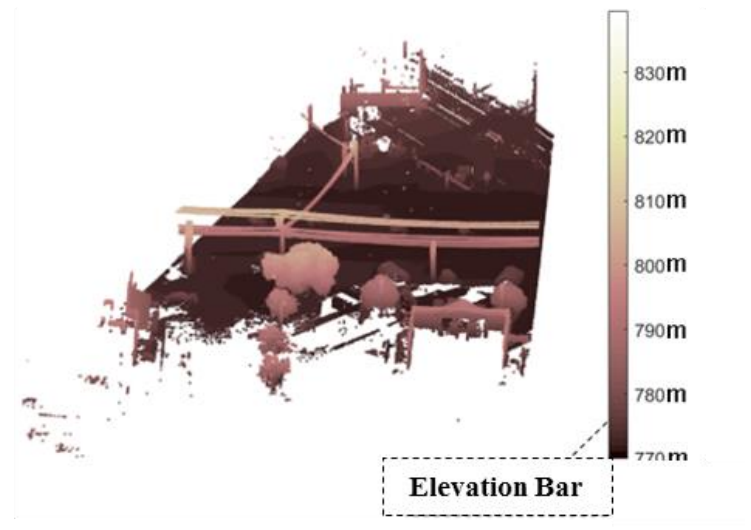

(b)

Figure 8. Noise removal of the point clouds with abnormal elevation. (a) A sample section which includes noisy points; (b) Removing the noisy points from the considered section.

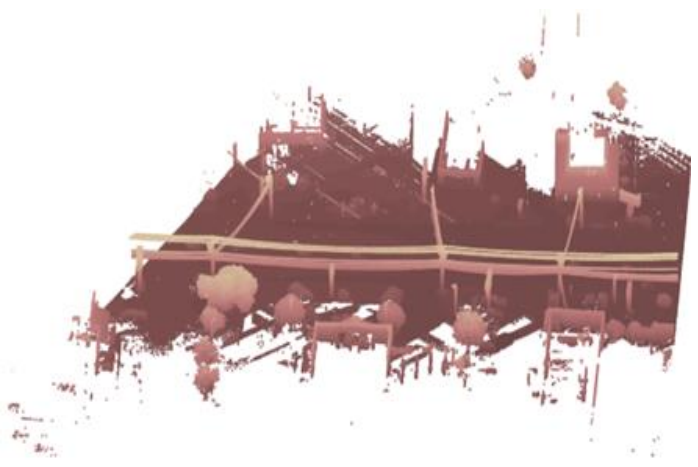

(a)

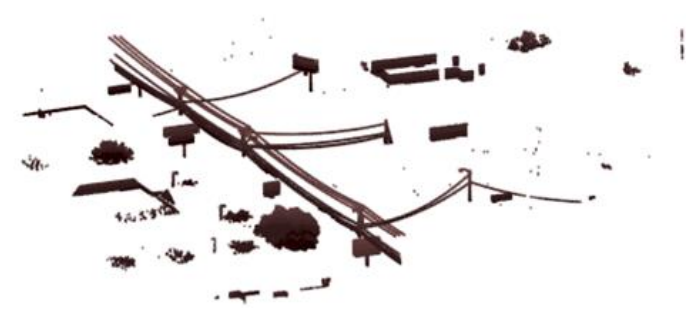

(b)

Figure 9. Low-Height Filtering step in order to eliminate unneeded objects. (a) A sample of the created sections; (b)

Eliminating unneeded objects such as ground points. 
In the cable extraction step, the pixel size of $30 \mathrm{~cm}$ was considered to create raster images. Figure 10-a shows the obtained raster image for the sample section. The raster images were enhanced using the Closing morphological operator. The Canny algorithm was applied to extract the edges on the image. The step-by-step output of the HT algorithm on this binary image is presented by Figures 10-b, 10-c and 10-d. As can be seen from these figures, the HT algorithm has successfully detected the cable area detection.

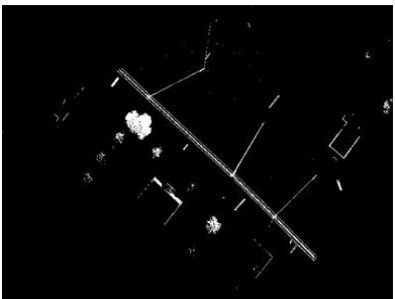

(a)

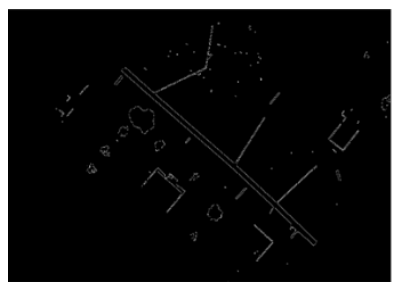

(c)

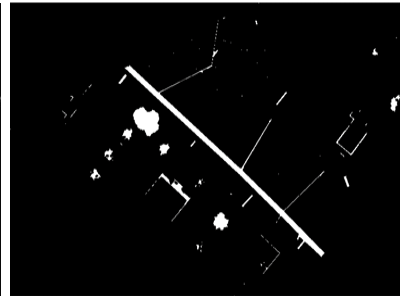

(b)

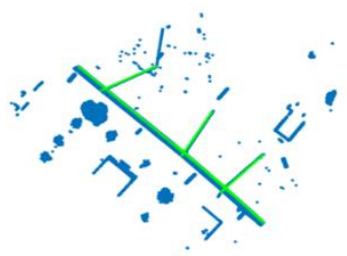

(d)
Figure 10. Cable area detection using the HT algorithm. (a) Creating a binary image from the remaining point cloud after low-height objects filtering; (b) Applying closing morphological operator to enhance the binary image; (c) Edge detection by canny algorithm; (d) Detected lines with the HT algorithm that are transferred back to the point cloud space.

The interval space for point sampling in the horizontal density profiling was $0.1 \mathrm{~m}$, and a $0.4 \mathrm{~m} \times 2 \mathrm{~m}$ rectangle perpendicular to the obtained cable area in each sampling point was considered as the neighboring window for calculating the density. Figure 11 shows an example of the horizontal density profiling.

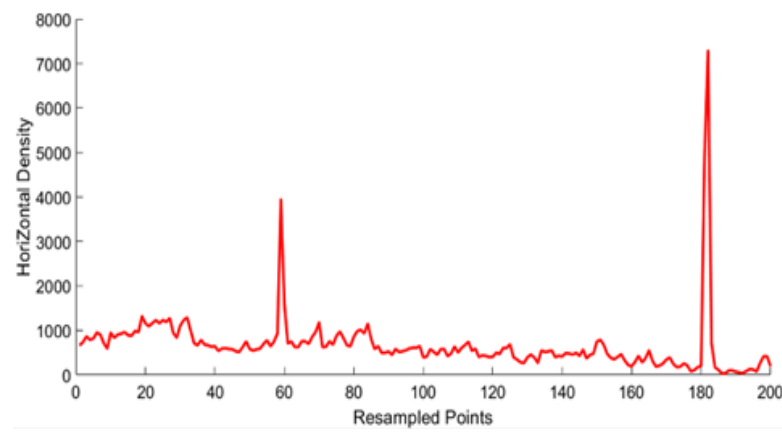

Figure 11. Diagram of horizontal density of a line which located on a cable area.

Also, the vertical density profile for each candidate pole was obtained with a $0.8 \mathrm{~m}$ sampling interval along the $\mathrm{Z}$ axis (Figure 12). In this step, the first condition to accept the candidate pole as a true pole was that the density in of all cubes should be greater than zero. Also, the threshold for the standard deviation was considered 4.5 .

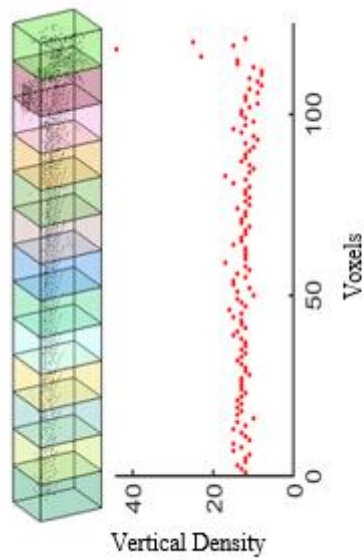

(a)

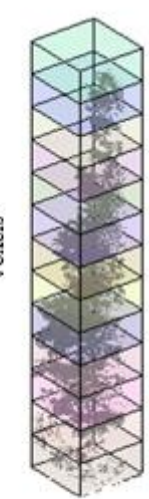

(b)

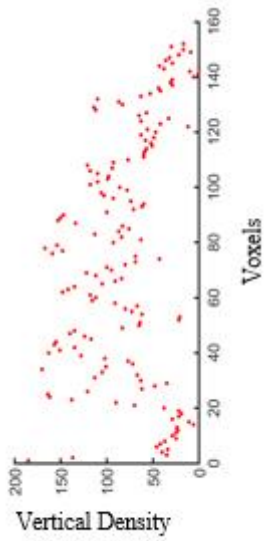

b)
Figure 12. Illustrating diagram of vertical density of a pole and non-pole object. (a) Vertical density of a pole object. (b)

Vertical density of a non-pole object.

Finally, all the utility poles were extracted from the dataset. Figure 13 displays the final output from the proposed algorithm. In the study area a total of 50 utility poles were manually identified and the proposed method successfully and correctly extracted 49 poles with no false positives.

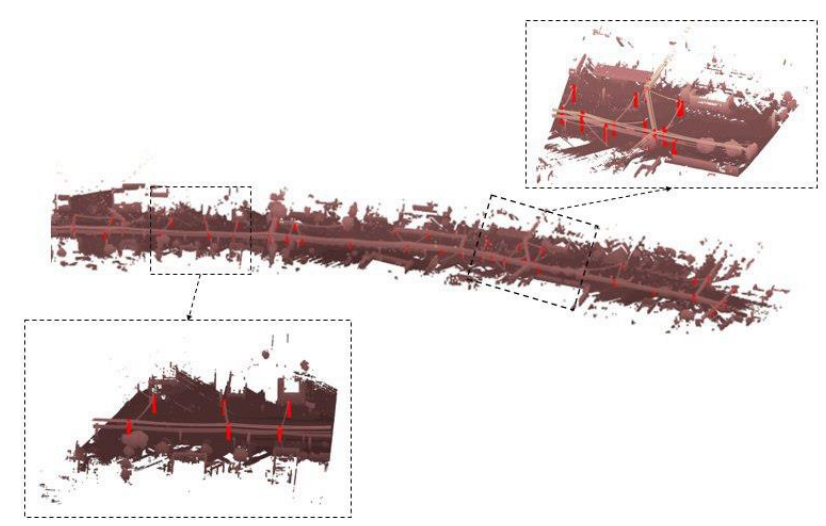

(a)
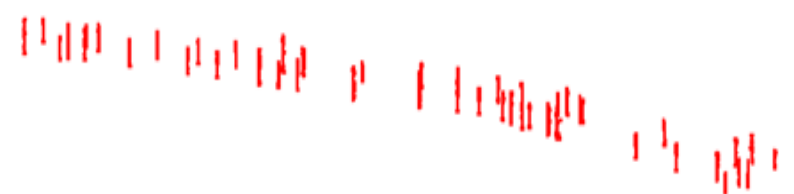

(b)

Figure 13. Output of the proposed algorithm which identified by red color. The proposed method successfully extracted 49 poles among 50 poles available at the urban area.

\subsection{Discussion}

The proposed method was evaluated in an urban area which the authors believe is more challenging in comparison to a nonurban area used in most previous studies. This is because rural areas do not usually have pole like features (other than trees) such as light poles. The focus in this research was extracting only utility poles. An extracted accuracy of $98 \%$ was achieved and there were no non-utility poles included in the final output (false positives). It should be noted that the only information used in this method are density and trajectory data which shows the superiority of the proposed method compared with methods that 
require acquired images, intensity information, and scan angle (Zia et al., 2013; Yang et al., 2015; Yadav and Chousalkar, 2017) which may not be available. Moreover, in our method the manually collecting training data, which is a time-consuming process, is not necessary. The proposed method was implemented in MATLAB and tested on a laptop system with normal configuration of Intel (R) Core (TM) i5-3210M CPU @ 2.50GHz, 12GB RAM, DDR 3, NVidia GeForce 2.630 GB, and does not need a cloud computing system with 36-Cores, 128GB DDR4 RAM which used in Yadav et al., (2016).

\section{CONCLUSION AND FUTURE WORKS}

This paper proposed a method for extracting utility poles from MTLS point clouds by initially dividing the data into smaller sections and eliminating noisy and unneeded pointes from each section. The HT algorithm is used to detect cable areas associated with utility poles, and then only utility poles are extracted based on density information. The proposed method was tested on a sample point cloud data set from an urban corridor that includes vehicle traffic, signs, light poles, buildings, trees, and other planimetric features. The results showed $98 \%$ accuracy in detecting utility poles. High speed and easy-to-implement in addition to using only the trajectory and density information can be the most important strengths of the proposed method. Although the results were promising, testing the proposed method on other datasets is desirable. Applying the method to airborne LiDAR may allow collection of utility poles in locations where MTLS is not feasible. Processing an airborne LiDAR set presents additional challenges because of the collection angle.

\section{REFERENCES}

Bae, S., Reineking, B., Ewald, M., Mueller, J., 2014. Comparison of airborne lidar, aerial photography, and field surveys to model the habitat suitability of a cryptic forest species - the hazel grouse. International Journal of Remote Sensing 35, 6469-6489.

Baffour, R.A., 2002. Collecting Roadway Cross Slope Data Using Multi-Antenna-Single Receiver GPS Configuration Seventh International Conference on Applications of Advanced Technologies in Transportation (AATT)

Balado, J., Díaz-Vilariño, L., Arias, P., Soilán, M., 2017. Automatic building accessibility diagnosis from point clouds. Automation in Construction 82, 103-111.

Cabo, C., Ordoñez, C., García-Cortés, S., Martínez, J., 2014. An algorithm for automatic detection of pole-like street furniture objects from Mobile Laser Scanner point clouds. ISPRS Journal of Photogrammetry and Remote Sensing 87, 47-56.

Che, E., Jung, J., Olsen, M.J., 2019. Object Recognition, Segmentation, and Classification of Mobile Laser Scanning Point Clouds: A State of the Art Review. Sensors 19, 810.

Chen, X., Wang, S.a., Zhang, B., Luo, L., 2018. Multi-feature fusion tree trunk detection and orchard mobile robot localization using camera/ultrasonic sensors. Computers and Electronics in Agriculture 147, 91-108.

Cui, H., Ren, X., Mao, Q., Hu, Q., Wang, W., 2019. Shield subway tunnel deformation detection based on mobile laser scanning. Automation in Construction 106, 102889.
Duda, R.O., Hart, P.E., 1972. Use of the Hough transformation to detect lines and curves in pictures, Communications of the ACM.

Guan, H., Yu, Y., Li, J., Ji, Z., Zhang, Q., 2016. Extraction of power-transmission lines from vehicle-borne lidar data. International Journal of Remote Sensing 37, 229-247.

Hao, W., Wang, Y., Li, Y., Shi, Z., Zhao, M., Liang, W., 2018. Hierarchical extraction of pole-like objects from scene point clouds. Optical Engineering 57, 1-11, 11 .

Holopainen, M., Kankare, V., Vastaranta, M., Liang, X., Lin, Y., Vaaja, M., Yu, X., Hyyppä, J., Hyyppä, H., Kaartinen, H., Kukko, A., Tanhuanpää, T., Alho, P., 2013. Tree mapping using airborne, terrestrial and mobile laser scanning - A case study in a heterogeneous urban forest. Urban Forestry \& Urban Greening $12,546-553$.

Jayathunga, S., Owari, T., Tsuyuki, S., 2018. Evaluating the Performance of Photogrammetric Products Using Fixed-Wing UAV Imagery over a Mixed Conifer-Broadleaf Forest: Comparison with Airborne Laser Scanning. Remote Sensing 10.

Kang, Z., Yang, J., Zhong, R., Wu, Y., Shi, Z., Lindenbergh, R., 2018. Voxel-Based Extraction and Classification of 3-D PoleLike Objects From Mobile LiDAR Point Cloud Data. IEEE Journal of Selected Topics in Applied Earth Observations and Remote Sensing 11, 4287-4298.

Landa, J., Ondroušek, V., 2016. Detection of Pole-like Objects from LIDAR Data. Procedia - Social and Behavioral Sciences 220, 226-235.

Lehtomäki, M., Jaakkola, A., Hyyppä, J., Kukko, A., Kaartinen, H., 2010. Detection of Vertical Pole-Like Objects in a Road Environment Using Vehicle-Based Laser Scanning Data. Remote Sensing 2.

Lehtomäki, M., Kukko, A., Matikainen, L., Hyyppä, J., Kaartinen, H., Jaakkola, A., 2019. Power line mapping technique using all-terrain mobile laser scanning. Automation in Construction 105, 102802.

Li, F., Lehtomäki, M., Elberink, S.O., Vosselman, G., Puttonen, E., Kukko, A., Hyyppä, J., 2018. Pole-like road furniture detection in sparse and nevenly distributed mobile laser scanning data, ISPRS Annals of the Photogrammetry, Remote Sensing and Spatial Information Sciences. ISPRS TC II Mid-term Symposium "Towards Photogrammetry 2020, Riva del Garda, Italy.

Li, F., Lehtomäki, M., Oude Elberink, S., Vosselman, G., Kukko, A., Puttonen, E., Chen, Y., Hyyppä, J., 2019a. Semantic segmentation of road furniture in mobile laser scanning data. ISPRS Journal of Photogrammetry and Remote Sensing 154, 98 113.

Li, Y., Wang, W., Tang, S., Li, D., Wang, Y., Yuan, Z., Guo, R., Li, X., Xiu, W., 2019b. Localization and Extraction of Road Poles in Urban Areas from Mobile Laser Scanning Data. Remote Sensing 11. 
Ordóñez, C., Cabo, C., Sanz-Ablanedo, E., 2017. Automatic Detection and Classification of Pole-Like Objects for Urban Cartography Using Mobile Laser Scanning Data. Sensors 17.

Otsu, N., 1979. A Threshold Selection Method from Gray-Level Histograms. IEEE Transactions on Systems, Man, and Cybernetics 9, 62-66.

Riveiro, B., González-Jorge, H., Martínez-Sánchez, J., DíazVilariño, L., Arias, P., 2015. Automatic detection of zebra crossings from mobile LiDAR data. Optics and Laser Technology 70, 63-70.

Rusu, R.B., Marton, Z.C., Blodow, N., Dolha, M., Beetz, M., 2008. Towards 3D Point cloud based object maps for household environments. Robotics and Autonomous Systems 56, 927-941.

Serna, A., Marcotegui, B., 2014. Detection, segmentation and classification of 3D urban objects using mathematical morphology and supervised learning. ISPRS Journal of Photogrammetry and Remote Sensing 93, 243-255.

Shams, A., Sarasua, W.A., Famili, A., Davis, W.J., Ogle, J.H., Cassule, L., Mammadrahimli, A., 2018. Highway Cross-Slope Measurement Using Mobile LiDAR. Transportation Research Record 39, 88-97.

Souleyrette, R., S, Hallmark, S., Patnaik, M., O'Brien, Veneziano, D., 2003. Grade and Cross Slope Estimation from LIDAR based Surface Models. U.S. Department of Transportation Research and Special Programs Administration.

Wen, C., Sun, X., Li, J., Wang, C., Guo, Y., Habib, A., 2019. A deep learning framework for road marking extraction, classification and completion from mobile laser scanning point clouds. ISPRS Journal of Photogrammetry and Remote Sensing 147, 178-192.

Xu, S., Xu, S., Ye, N., Zhu, F., 2018. Automatic extraction of street trees' nonphotosynthetic components from MLS data. International Journal of Applied Earth Observation and Geoinformation 69, 64-77.

Yadav, M., Chousalkar, C.G., 2017. Extraction of power lines using mobile LiDAR data of roadway environment. Remote Sensing Applications: Society and Environment 8, 258-265.

Yadav, M., Lohani, B., Singh, A.K., Husain, A., 2016. Identification of pole-like structures from mobile lidar data of complex road environment. International Journal of Remote Sensing 37, 4748-4777.

Yang, B., Dong, Z., Zhao, G., Dai, W., 2015. Hierarchical extraction of urban objects from mobile laser scanning data. ISPRS Journal of Photogrammetry and Remote Sensing 99, 4557.

Yang, B., Wei, Z., Li, Q., Li, J., 2012. Automated extraction of street-scene objects from mobile lidar point clouds. International Journal of Remote Sensing 33, 5839-5861.

Ye, F., Li, F., Hu, S., 2014. Automatic extraction of power lines by particle filtering from aerial images. SPIE.
Yokoyama, H., Date, H., Kanai, S., Takeda, H., 2011. Pole-Like Objects Recognition from Mobile Laser Scanning Data Using Smoothing and Principal Component Analysis, ISPRS International Archives of the Photogrammetry, Remote Sensing and Spatial Information Sciences. ISPRS, Calgary, Canada, pp. 115-120.

Yu, X., Hyyppä, J., Karjalainen, M., Nurminen, K., Karila, K., Vastaranta, M., Kankare, V., Kaartinen, H., Holopainen, M., Honkavaara, E., Kukko, A., Jaakkola, A., Liang, X., Wang, Y., Hyyppä, H., Katoh, M., 2015a. Comparison of Laser and Stereo Optical, SAR and InSAR Point Clouds from Air- and SpaceBorne Sources in the Retrieval of Forest Inventory Attributes. Remote Sensing 7.

Yu, Y., Guan, H., Ji, Z., 2015b. Automated Detection of Urban Road Manhole Covers Using Mobile Laser Scanning Data. IEEE Transactions on Intelligent Transportation Systems 16, 3258 3269.

Zia, M.Z., Stark, M., Schiele, B., Schindler, K., 2013. Detailed 3D Representations for Object Recognition and Modeling. IEEE Transactions on Pattern Analysis and Machine Intelligence 35, 2608-2623. 